\title{
Breast cancer - one term, many entities?
}

\author{
Nicholas R. Bertos ${ }^{1,2}$ and Morag Park',2,3,4 \\ ${ }^{1}$ Rosalind and Morris Goodman Cancer Research Centre, ${ }^{2}$ Department of Medicine, and ${ }^{3}$ Departments of Oncology and Biochemistry, \\ McGill University, Montreal, Quebec, Canada. ${ }^{4}$ Department of Medicine, McGill University Health Centre, Montreal, Quebec, Canada.
}

\begin{abstract}
Breast cancer, rather than constituting a monolithic entity, comprises heterogeneous tumors with different clinical characteristics, disease courses, and responses to specific treatments. Tumor-intrinsic features, including classical histological and immunopathological classifications as well as more recently described molecular subtypes, separate breast tumors into multiple groups. Tumor-extrinsic features, including microenvironmental configuration, also have prognostic significance and further expand the list of tumor-defining variables. A better understanding of the features underlying heterogeneity, as well as of the mechanisms and consequences of their interactions, is essential to improve targeting of existing therapies and to develop novel agents addressing specific combinations of features.
\end{abstract}

\section{Normal architecture of the breast}

To understand how such variation arises, it is instructive to examine the normal architecture of the breast environment (Figure 1A). Each lobe arises from multiple lobules, which connect to a common terminal interlobular duct. These ducts then continue to their outlet at the nipple. Histologically, lobules and ducts are lined by a single layer of luminal epithelial cells, surrounded by transversely oriented myoepithelial cells. These structures are separated from the surrounding tissue, or stroma, by a basement membrane, the breach of which distinguishes invasive carcinoma from carcinoma in situ (1). The surrounding stroma comprises ECM, discrete cells (e.g., fibroblasts, immune cells, and adipocytes), and organized structures (e.g., blood vessels), each of which contributes to the overall configuration of the local microenvironment.

\section{Structural heterogeneity}

Classical pathology has segregated breast tumors into multiple categories, based on their overall morphology and structural organization. The most common type observed and reported is invasive ductal carcinoma, not otherwise specified (IDC NOS; about $75 \%$ of cases), while invasive lobular carcinoma (ILC) represents the next most frequent histologic type of breast tumor (about $10 \%$ of cases) (2). Together, these two categories and combinations thereof make up the vast majority (about $90 \%$ ) of breast cancers, while the remainder are categorized as medullary, neuroendocrine, tubular, apocrine, metaplastic, mucinous (A and B), inflammatory, comedo, adenoid cystic, and micropapillary types $(2,3)$. Interestingly, histologic type is linked to prognosis. While IDC NOS, ILC, apocrine, and medullary carcinomas have similar 10-year survival rates, adenoid cystic, medullary, mucinous, and tubular carcinomas exhibit relatively better overall outcomes (4-6). However, the rarity of specific non-ILC/non-IDC tumors (known as "special types") has resulted in a relative paucity of in-depth characterization of larger cohorts of such cases, and thus the details of how membership in these groups interacts with other factors contributing to tumor heterogeneity are not well understood.

\section{Immunopathological classifications}

The presence of specific markers in breast cancer has long been recognized to both define subtypes with differential overall

Conflict of interest: The authors have declared that no conflict of interest exists. Citation for this article: J Clin Invest. 2011;121(10):3789-3796. doi:10.1172/JCI57100. prognosis and to identify tumors susceptible to targeted treatments. The chief markers assessed are estrogen receptor (ER), progesterone receptor (PR), and human epidermal receptor 2 (HER2). Expression of the first two is assayed almost exclusively by immunohistochemistry (IHC)-based methods, which report levels of the corresponding proteins, while HER2 assays combine IHC and FISH approaches. Ambiguous IHC-derived HER 2 results are subjected to FISH testing for genomic amplification of HER2; cases in which the overall ratio of copies of the HER2 gene to those of its chromosome is greater than 2.2 are reported as HER2 ${ }^{+}$(7). ER status is utilized to identify tumors that may respond to anti-estrogen (endocrine) therapy, including ER antagonists or aromatase inhibitors, which target ER-dependent signaling $(8,9)$. PR status, generally correlated with ER status, has less clinical significance. PR status does not appear to predict relative benefit from specific types of endocrine therapy $(10,11)$, and overall, $\mathrm{ER}^{+} / \mathrm{PR}^{+}$cases may not receive additional benefit from endocrine therapy compared with $\mathrm{ER}^{+} / \mathrm{PR}^{-}$cases $(12-14)$. HER2 ${ }^{+}$cases are treated with targeted therapies such as the monoclonal antibody trastuzumab, which binds to HER2, mediates antibody-dependent cytotoxicity, and disrupts HER2-dependent signaling $(15,16)$. There is currently no standard targeted therapy for cases assessed as ER- and HER- by IHC, although this represents an intensive area of research.

Combinations of these markers allow for the assignment of individual cases to specific categories, namely $\mathrm{ER}^{+}\left(\mathrm{ER}^{+} / \mathrm{HER} 2^{-}\right)$, $\mathrm{HER}^{+}{ }^{+} \mathrm{ER}^{-} / \mathrm{HER} 2^{+}$), triple negative (TN; ER $-/ \mathrm{PR}^{-} / \mathrm{HER} 2^{-}$), and triple positive $\left(\mathrm{ER}^{+} / \mathrm{PR}^{+} / \mathrm{HER} 2^{+}\right)$. From a prognostic viewpoint, $\mathrm{ER}^{+}$tumors exhibit the best overall outcome. Following the advent of HER2-targeted therapies, HER2 ${ }^{+}$tumors, previously associated with poor outcome, now exhibit an improved overall outcome when treated with such therapies $(17,18)$. TN tumors, on the other hand, are linked to the worst prognosis among the subtypes (19), while triple-positive cases appeared to have a prognosis intermediate between those of $\mathrm{ER}^{+}$and $\mathrm{HER} 2^{+}$cases prior to the introduction of HER2-targeted treatments (20).

\section{Intratumoral heterogeneity}

It is important to note that heterogeneity within individual tumors is significantly more prevalent than the status assignments by ER-, PR-, and HER2-directed assays described above would indicate. For example, $\mathrm{ER}^{+}$status is currently reported when the proportion of $\mathrm{ER}^{+}$tumor cells within the tumor exceeds a $1 \%$ threshold (21), while the IHC threshold for HER2 positivity is met when more than $30 \%$ 
A

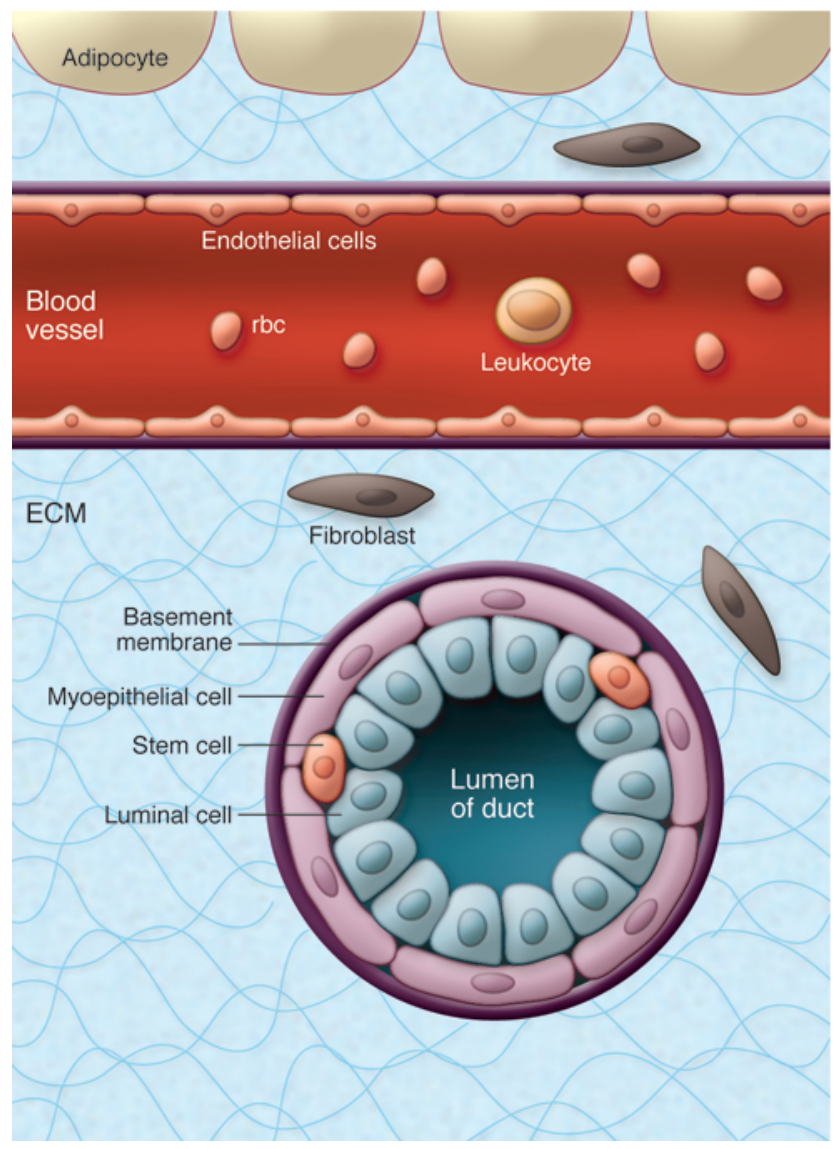

B

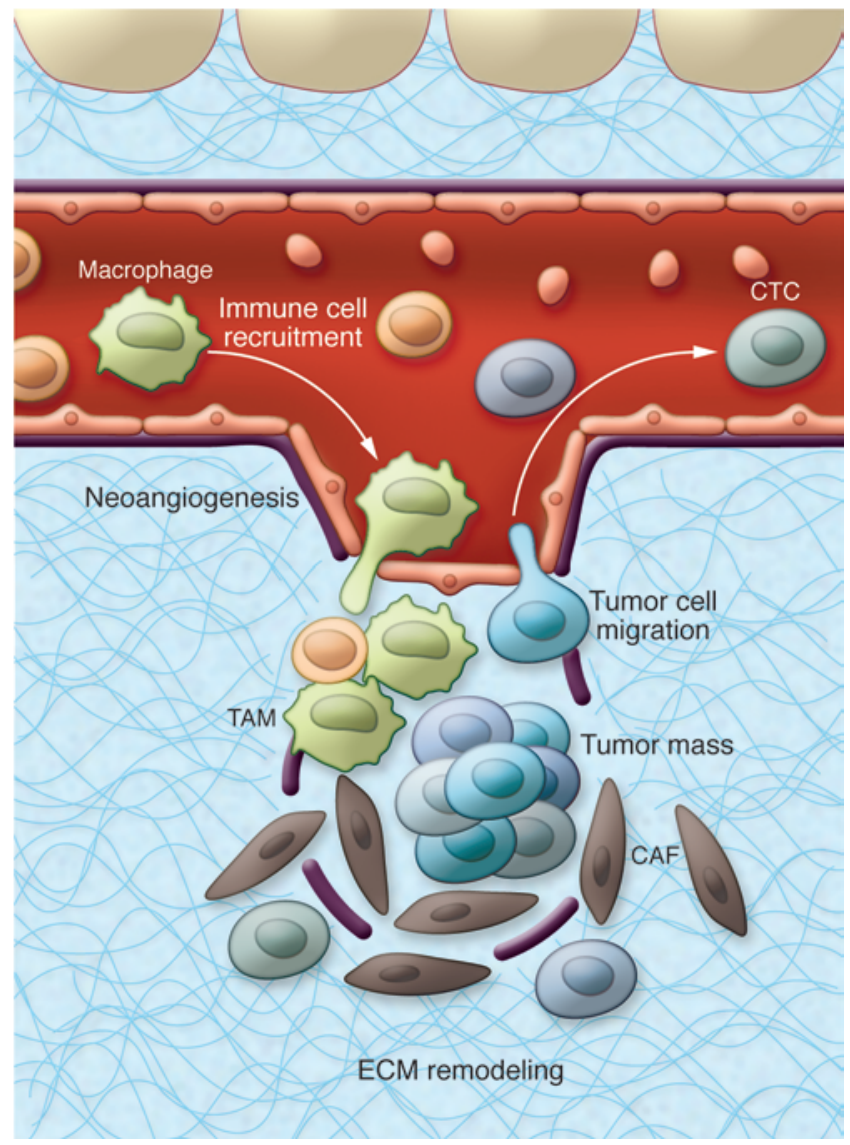

Figure 1

Schematic depiction of normal breast architecture and breast tumor and surrounding stroma, illustrating some of the tumor-intrinsic and microenvironmental variables contributing to disease heterogeneity. (A) Normal breast architecture. (B) Breast tumor and surrounding stroma. TAM, tumor-associated macrophage.

of cells display strong membrane-associated HER2 expression. These low thresholds necessarily imply that in many cases, the majority of the tumor cells present display features inconsistent with the overall status assigned. In addition, the clinical course of disease may be governed by alterations occurring within relatively small subsets of primary tumor cells. This is reflected by differences between the primary tumor and metastatic lesions at the ER/HER2 status (22) and genomic (23) levels. In this context, it is important to note that reporting thresholds vary over time and between laboratories (e.g., the threshold for ER positivity has changed from $10 \%$ to $1 \%$ of tumor cells exhibiting ER positivity in IHC analysis; ref. 24). Thus, conclusions and correlations based on reported receptor status from different studies may not be directly comparable (25).

Significant intratumoral heterogeneity also exists at the genomic level. Investigation of discrete tumor regions by comparative genomic hybridization established that significant genomic heterogeneity was present in more than half (5 of 9) of all tumors examined (26). Studies of genomic alterations in multiple single cells from individual tumors have revealed that over half of the samples examined are polygenomic, containing multiple clonal subpopulations that may be spatially separated or intermixed $(27,28)$. Future studies will be required to address the implication of these observations.

\section{Molecular heterogeneity at the gene expression level}

Over the past decade, the advent of high-throughput/high-content microarray-based gene expression profiling technologies has facilitated relatively large-scale studies of breast cancer cohorts, leading to the identification of multiple molecular subtypes of breast cancer. Studies by Perou and colleagues (29-32) described molecular subtypes defined by distinct transcriptional signatures that partially recapitulate the original immunopathological classes, while adding an additional level of detail. Two luminal subtypes (A and B) contain principally $\mathrm{ER}^{+}$cases and are distinguished by the presence of genes regulated by the ER signaling pathway. The luminal A subtype is associated with higher levels of ESR1, ER, and ER-regulated genes (32), decreased proliferation (29, 33, 34), and improved overall outcome $(29,30,32)$. Luminal B tumors appear to exhibit decreased levels of ESR1, ER, and ER-regulated genes as well as increased proliferation and relatively worse prognosis.

Other classification schemes have also been proposed, including one that divides $\mathrm{ER}^{+}$disease into four subtypes that are chiefly distinguished by differential expression of proliferation-related genes (35). Recent investigations suggest that the distribution of luminal cases, rather than forming two distinct categories, may be better modeled as a continuum along which ER-regulated elements and proliferation are inversely related 
$(36,37)$. This model is further supported by the finding that gene expression signatures designed to classify novel samples by molecular subtype $(29,38,39)$ exhibit substantial disagreement for luminal cases $(40,41)$, implying that the luminal subtypes are not as distinct as initially believed.

In the scheme proposed by Perou and colleagues, three subtypes contain predominantly ER ${ }^{-}$cases (31). The molecular ERBB2+ subtype generally (but imperfectly) overlaps with IHC-defined HER2 ${ }^{+}$tumors (31), while the basal or basal-like subtype broadly corresponds to the TN (ER-/PR-/HER2-) cohort (42, 43). A normal-like molecular subtype resembles normal epithelial tissue (44) and may comprise cases in which samples contain large amounts of non-tumor tissue $(29,39)$. In an alternate scheme, two ER- subtypes are defined by different expression levels of the androgen receptor, while HER2 ${ }^{+}$cases do not form a separate group, but rather cluster according to their ER status (35). Other molecular subtypes within the $\mathrm{ER}^{-}$cohort have also been reported. These include a claudin-low subtype (45) linked to metaplastic breast cancer and poor outcome, which shows similarity to stem celllinked and epithelial-to-mesenchymal transition-linked (EMTlinked) signatures, and the molecular apocrine class, enriched in $\mathrm{ER}^{-} / \mathrm{HER} 2^{+}$tumors and displaying positivity for androgen receptor and downstream signaling (46).

TN breast cancers are necessarily defined by the absence of specific markers, suggesting that significant heterogeneity may exist within this group. An investigation of expression profiles from $587 \mathrm{TN}$ breast tumors further subdivided these into 6 groups (47). These exhibited preferential responses to specific chemotherapeutic regimens, as well as differential expression of basal-specific, immunomodulatory, mesenchymal, mesenchymal stem-like, and androgen receptor-related genes, likely reflecting several of the classes discussed above.

Clustering by its nature leads to definitive assignments of tumors to specific subtypes. However, individual tumors likely display combinations of features of different subtypes, whether due to intratumoral heterogeneity or to a mixed phenotype of individual cells. Thus, the specific nature of a tumor may be better defined by its location within a multidimensional continu$\mathrm{um}$, in which the canonical subtype-defining signatures represent vertices, than by a single label. It is important to note that gene expression profiles derived from bulk tumor by definition must reflect an average value of the cells surveyed. The fact that these profiles nevertheless significantly correlate with disease course suggests that they may reflect the propensity of tumors exhibiting distinct processes, e.g., proliferation, to give rise to subpopulations capable of metastasis.

\section{Genomic heterogeneity}

The principal molecular subtypes were each found to be associated with specific patterns of genomic alterations (48-53). Six genomic subtypes have been identified in breast cancer. Four of these overlap with gene expression-defined groups (ERBB2 ${ }^{+}$, basal-like, luminal); however, the overall luminal cohort was imperfectly segregated between luminal A and luminal B cases, while two genomic classes (amplifier and mixed) contained tumors from multiple gene expression-defined subtypes (54). These results further suggest that gene expression-derived classifications may not fully define the spectrum of diversity present across breast tumors. Certain genomic changes, e.g., amplification of the HER2-containing amplicon, are clearly linked to the molecular features of the cor- responding tumor subtype. However, the extent to which genomic alterations determine features of tumor subtype, or whether they also partially reflect variations in mechanisms of genomic instability between subtypes, remains an open question.

A new area for studying the behavior of breast cancers has arisen with the discovery of microRNAs (miRNAs), short RNA molecules (approximately 22 nucleotides) that play roles in transcriptional and posttranscriptional regulation of gene expression $(55,56)$. Analyses of miRNA profiles in breast cancer have determined that many miRNAs display expression patterns linked to molecular subtype (57-60) as well as ER status, tumor grade (58), and other tumor-related processes (61-63). Experimental evidence has confirmed that miRNA levels can play a role in determining disease course; for example, re-expression of miRNA-193b, downregulated in highly metastatic derivatives of the MDA-MB-231 cell line, significantly inhibited tumor growth and dissemination in a mouse xenograft model (64). Thus, integrated analysis of miRNA and mRNA profiles in breast cancer constitutes an important new frontier $(60,65)$.

While some changes in miRNA expression are correlated with genomic changes or local alterations in primary transcription rates, changes in overall miRNA biogenesis may also underlie alterations of miRNA levels (58). Transcriptional levels of the miRNA-processing elements $A G O 2$ and DICER1 correlate with ER status, proliferation status, tumor molecular subtype, and disease outcome (58, 66-68), while decreased levels of the miRNA processor Drosha are linked to HER2 positivity (67).

Many of the mRNA- and miRNA-level alterations observed in breast cancer cannot be ascribed to genomic changes. These are likely linked to epigenetic factors, including DNA methylation and histone modifications. DNA methylation within the ESR1-containing CPG island was increased in ER- breast cancer samples compared with $\mathrm{ER}^{+}$samples (69-71), suggesting that methylation-induced ESR1 silencing may govern ER expression in some cases. Recent studies including samples from different molecular subtypes demonstrate that characteristic differential DNA methylation profiles subdivide samples into multiple luminal A-enriched and basal-like/ERBB2+-enriched clusters $(72,73)$. Interestingly, methylation cluster-specific survival differences were observed within luminal A samples (73), suggesting that additional information beyond molecular subtype alone is captured by methylation profiling.

\section{Differences in cell of origin}

The expression patterns of specific cytokeratins (CKs) in the luminal and basal molecular breast cancer subtypes resemble those of normal luminal epithelial (CK8/18-expressing) and basal stem (CK5/6-expressing) cells of the breast, respectively. Therefore, it has been proposed that these tumors arise from different cells of origin, and that luminal tumors arise from luminal progenitor cells, while basal tumors originate from the basal or stem cell compartment $(38,74,75)$. However, comparisons of the transcriptional signatures of the molecular breast cancer subtypes with gene expression profiles of cell subsets isolated from mammary tissue suggest that different molecular subtypes of breast cancer may arise from cells at various stages of differentiation along the mammary epithelial hierarchy (76).

Claudin-low tumors display gene expression profiles similar to those of mammary stem cells, while basal tumors resemble bipotent or early luminal progenitors (77-79). ERBB2+ tumors are 
most similar to late luminal progenitors, while luminal tumors are closest to the differentiated luminal cell compartment (76). This scheme is somewhat complicated by the possibility that tumor cells may drift from their original configuration, losing specific markers of the cell of origin and taking on others (epithelial plasticity). A prime example of this is EMT, in which transformed epithelial cells switch to a mesenchymal phenotype whose expression profile resembles that of stem cells (80). EMT is associated with increased motility and enhanced invasion. However, once they reach a distant site, these cells can then revert to an epithelial phenotype following interactions with the local microenvironment (81-84).

\section{Tumors of special types}

The principal studies reporting the diversity of molecular breast cancer subtypes were conducted using samples from the most common histological subtypes, i.e., IDC and occasionally ILC tumors. Tumors of special types, i.e., non-IDC NOS cases, constitute $25 \%$ of breast tumors, generally display high uniformity with respect to ER and HER2 status within each type (85), and are commonly associated with good clinical outcome (86). Microarray-based gene expression profiling of small cohorts of breast tumors of special types category established that special types tend to cluster within single subtypes (87), while IDC NOS tumors display a variety of molecular subtypes. For example, tubular carcinomas and standard ILC samples display similar gene expression patterns and fall into the luminal category, while pleomorphic ILC samples group with apocrine tumors as members of the non-luminal molecular apocrine molecular subtype. However, $\mathrm{ER}^{+}$micropapillary and mucinous tumors have gene expression profiles distinct from those $\mathrm{ER}^{+}$ IDC (NOS) samples (88-90). Neuroendocrine and mucinous A and B samples, considered to be histologically distinct entities, cluster together within the luminal subdivision (87), although mucinous A tumors form a distinct subgroup (91). Interestingly, other ER- tumors, including members of the adenoid cystic, medullary, and metaplastic histological subtypes, are highly similar (87). In some cases, this homogeneity is likely due at least partially to underlying common features; for example the $M Y B-N F I B$ fusion was found in adenoid cystic carcinomas (4 of 4 cases) (92), while the extremely rare secretory carcinomas harbored an ETV6-NTRK3 gene fusion (12 of 13 cases) (93). These findings suggest that the histological features defining members of special types reflect specific underlying molecular configurations, unlike what is seen in the case of IDC NOS tumors.

\section{Microenvironmental heterogeneity}

Recent studies have demonstrated that disease course is not solely linked to tumor-intrinsic features, but that features of the local microenvironment, or stroma, can strongly influence outcome (94-100). Patient-specific variations in the abundance and status of different stromal cell types, as well as communication between and mutual modulation of each compartment (tumor and stroma), induce additional levels of complexity and contribute to breast cancer heterogeneity and disease course (Figure 1B). Stromal elements contributing to this heterogeneity include the ECM itself, alterations of which have been proposed to contribute to tumorigenesis (101) and response to treatment (102). A mouse model with increased stromal type I collagen exhibited increased tumor formation and invasion (103), likely via a $\beta 1$-integrin-dependent mechanism (104), while normal, but not tumor-associated, myoepithelial cells reversed invasion in DCISlike cells via synthesis of laminin-1 (105). While myoepithelial cells are commonly absent in breast tumors, the interactions described above likely influence early stages of disease initiation and progression to malignancy.

Immune cells, fibroblasts, and endothelial cells also vary in number and type among different tumors. Increased numbers of tumor-associated macrophages are associated with poor disease outcome (106), reflecting their ability to enhance tumor cell invasion (107-108). This is mediated in part via a mutual paracrine loop involving growth factors produced by macrophages that influence tumor cell migration, such as epidermal growth factor, and growth factors produced by tumor cells that attract macrophages, such as colony stimulating factor 1 (109). Tumorassociated $\mathrm{T}$ cells can have different effects, depending on activation status. Th1-type $\mathrm{T}$ cells are associated with good outcome (99, 110-113), while immunosuppressive Tregs are associated with tumor progression and poor prognosis (113-116), and response to neoadjuvant therapy correlates with changes in Treg numbers (117). In addition, microenvironmental factors, including $\mathrm{T}$ cell status, may play a more prominent role in determining outcome in ER- and HER2 $2^{+}$tumors $(99,118)$, while tumor proliferation is more closely correlated with outcome in $\mathrm{ER}^{+}$disease.

Carcinoma-associated fibroblasts (CAFs) are known to secrete tumor-promoting factors, including the chemokine SDF-1/ CXCL12, which promotes tumor cell migration into the stroma (119) and enhances angiogenesis through recruitment of endothelial progenitor cells (120), as well as matrix metalloproteinases, which can mediate degradation of the ECM (121). Interestingly, manipulation of stromal fibroblasts via specific ablation of the tumor suppressor gene PTEN, which negatively regulates the Akt kinase and cell survival and proliferation pathways, supports enhanced tumor development of a Neu/ErbB2-driven tumor xenograft (122). In contrast, fibroblast-specific ablation of the ETS2 transcription factor reduces Neu/ErbB2 tumor growth, demonstrating the functional importance of fibroblast-dependent interactions in disease outcome (122).

Angiogenesis is a necessary step for tumor growth, and microvessel density has weak prognostic value in breast cancer $(123,124)$, demonstrating that this element is also heterogeneously distributed. Thus, a range of potential microenvironmental states and responses further enhances the heterogeneity of breast cancer. The full spectrum of these combinations, and the nature and extent of their individual interactions with tumor-intrinsic determinants of disease course, remains to be investigated.

\section{Macroenvironmental heterogeneity}

Beyond those features of the stroma that are specific to the local tumor microenvironment, systemic factors may also contribute to the range of behaviors evinced by breast tumors. These include factors such as age, menopausal status, and variations in body mass index (the latter two affecting systemic estrogen and progesterone levels; ref. 125), as well as overall immune status. For example, obesity is associated with increased tumor recurrence and decreased survival (126-128) as well as with a distinct tumor transcriptional signature (129), potentially due to the influence of surrounding adipocytes on tumor tissue (126). How these variables interact with tumor-intrinsic and local microenvironmental features remains an area for further study. 


\section{Table 1}

Selected elements contributing to breast tumor heterogeneity

\author{
Classifier \\ Histological \\ Immunopathological \\ Transcriptional \\ Genomic \\ Genomic heterogeneity \\ miRNA-based \\ Epigenetic \\ Microenvironmental \\ Macroenvironmental \\ Longitudinal \\ Other
}

\author{
Classifications/variables \\ IDC NOS, ILC, medullary, neuroendocrine, tubular, apocrine, metaplastic, mucinous ( $A$ and $B$ ), \\ inflammatory, comedo, adenoid cystic, micropapillary \\ ER status, PR status, HER2 status \\ Luminal A, luminal B, normal-like, basal/basal-like, HER2, claudin low, molecular apocrine \\ 17q12, basal complex, luminal simple, luminal complex, amplifier, mixed \\ Monogenomic, polygenomic \\ Multiple \\ Multiple \\ Presence/activation status of local immune cells (T cells, B cells, dendritic cells, macrophages), \\ fibroblast status, ECM composition, CAF status, angiogenesis, hypoxia \\ Systemic hormone levels, BMI, overall immune status \\ CTC features, metastatic features \\ Intratumoral heterogeneity
}

\section{Longitudinal heterogeneity - alterations in tumor features during progression}

It has long been recognized that recurrences of breast cancer in the form of distant metastases may display characteristics that do not match those of the primary tumor. At the genomic level, metastases can present additional changes beyond those observed in the primary tumor (130), while discordance rates ranging from $13 \%$ to $54 \%$ have been reported for ER status and from $0 \%$ to $32 \%$ for HER2 status (22). Many of these discrepancies can be likely be ascribed to technical variation in sample preparation and testing or to sampling artifacts (since the entire primary lesion is not assessed). However, the high rates observed suggest that, in agreement with current models of tumor heterogeneity, metastases arise from subsets of cells present within the primary tumor; these subsets are not necessarily reflective of the overall tumor profile as reported either by molecular or IHC-based approaches. Thus, clinical management of disease must adapt to the changing and evolving nature of the tumor over time.

\section{Differential preference for sites of metastases}

Associations between classical subtypes as defined by receptor status and sites of distant metastasis have previously been reported (131). ER- disease preferentially metastasizes to the soft tissues and viscera (e.g., lungs, liver, brain), while distant recurrence of $\mathrm{ER}^{+}$breast cancer is more commonly seen in bone, and HER2+ tumors exhibit an increased rate of brain metastases. The molecular breast tumor subtypes also display distinct spectra of preferred metastatic sites. Brain metastases were relatively more common in basal-like and $\mathrm{ERBB}^{+}$disease, while recurrence in bone was more often observed in luminal (both A and B) and, to a lesser extent, ERBB2 $^{+}$tumors $(132,133)$. However, the HER2-targeting agent trastuzumab is unable to effectively cross the blood-brain barrier (134), potentially protecting HER $2^{+}$brain lesions.

It has been suggested that similarities between pathways activated within specific subtypes and those ordinarily active within the potential sites of metastasis may be important in determining preferred sites of distant spread (133), in accordance with the "seedand-soil" model originally proposed over a century ago (135). This suggested that metastases do not randomly target distant organs, but preferentially arise in those that offer the disseminated cancer cell an appropriate environment in which to grow or that express molecules supporting tumor cell homing. Recent studies demonstrate that the expression of specific molecules, including chemokine receptors as well as claudin-2, CCN3, and tenascin-C, correlate with and/or enhance metastasis to distinct sites (119, 136-138). The chemokine CXCL12 is expressed in bone marrow, and expression of its cognate receptor CXCR4 on breast tumor cells is correlated with an increased risk of metastasis to bone (139). Interestingly, cell lines selected for enhanced metastasis to bone display a specific transcriptional signature (including elevated CXCR4) that is also present in subsets of cells from the parental tumor cell population (140), suggesting that subpopulations of cells capable of metastasis to specific sites form part of the initial spectrum of heterogeneity in the primary tumor.

The "seed-and-soil" model may also apply to the local environment of the primary tumor. Tumor self-seeding, in which cells escaping from the primary tumor are hypothesized to later return to the same anatomical site and to be concentrated at the outer surface of the lesion (141-143), also leads to increased intratumoral heterogeneity and may contribute to the polygenomic nature of many tumors. This model supports a view of cancer progression as a process dependent on multiple factors beyond the local disease site. Support for this concept comes from a study demonstrating that indolent tumors are induced to grow by the presence of an actively proliferating tumor at an anatomically distinct site, and that this occurs via osteopontin-mediated mobilization and recruitment of bone marrow-derived cells into the stroma of the previously indolent tumor (144). Similarly, circulating estrogen can induce bone marrow-derived cell recruitment to the stroma of ER- tumors in a mouse model, leading to the promotion of tumor growth (145) - the recruitment of such cells further enhances heterogeneity in the tumor microenvironment.

\section{Circulating tumor cells}

In breast cancer, the majority of patients present with local disease, and the primary lesions are generally removed by surgery prior to the development of clinically detectable metastases. Therefore, prognostic information obtained through studies of the primary tumor must essentially reflect the relationship between features of this tissue and the likelihood that cells with the potential to proliferate at distant sites have already disseminated before primary tumor resection. In order to gain a better understanding of 
the elements governing recurrence, an intermediate between the primary tumor and distant metastases must be sought. Circulating tumor cells (CTCs) represent cells that have already escaped the primary tumor site and thus may be an appropriate candidate. These cells have a high level of agreement (82\%-89\%) at the level of HER2 status with the primary tumor $(146,147)$; however, concordances for ER and PR status are lower (41\% and $45 \%$, respectively) (148). These observations suggest that CTCs may act as a proxy for the subset of cells within the primary tumor capable of leading to disease recurrence. Interestingly, characterization of CTCs shows that subpopulations of these cells are enriched for stem cell and EMT markers $(149,150)$, suggesting that they arise from specific subgroups within the tumor. Further investigations of the correlation between markers of CTCs and those of distant metastases are expected to clarify the origin of CTCs and how their features influence disease course.

\section{Conclusions and future directions}

The identification of multiple sources of tumor heterogeneity at both tumor-intrinsic and -extrinsic levels, as well as the changes seen during progression (Table 1), suggests that an enhanced understanding of the effect of this heterogeneity on the impact of specific treatments represents an urgent clinical need. Therefore, it is desirable that provision is made within large-scale clinical trials for the collection of sufficient samples to assign tumors to specific combinations of classes. While markers for specific tumorintrinsic protein and transcript features either have already been included in standard clinical practice (e.g., IHC-based assessment of receptor status) or are beginning to approach standardization (i.e., the 50-gene PAM50 classifier for assignment to molecular subtypes), assays for microenvironmental features remain outside the realm of clinical use. Additional studies of large cohorts (including the histologic "special types" of tumors) at multiple concurrent levels, including integrated tumor-intrinsic, microenvironmental and macroenvironmental profiling, as well as analyses of matched CTCs and distant metastases and assessment of intratumoral heterogeneity, will be necessary. This will ultimately allow the development of a complete set of classifiers that, taken together, can define an individual tumor by its location in a multidimensional coordinate system comprising all of the variables contributing to breast cancer heterogeneity. Such schemes can then be applied to tissue and blood samples from clinical trials of specific therapies, generating an improved stratification system to predict the relative benefit of each potential intervention, or combinations thereof, in a model tailored to the specific combination of features defining an individual cancer.

\section{Acknowledgments}

Space constraints render it impossible to adequately acknowledge all of the contributions made to this field by the many key individuals and groups who have studied different aspects of this research area in detail, and in many cases have made it necessary to refer the reader to reviews. The authors apologize for any omissions. The authors acknowledge the valuable insights gained into breast tumor heterogeneity through discussions with colleagues from different fields at McGill University and the McGill University Health Centre, including Michael Hallett (Bioinformatics), Catalin Mihalcioiu (Medical Oncology), Sarkis Meterissian (Surgery), and Atilla Omeroglu (Pathology). M. Park holds the Diane and Sal Guerrera Chair in Cancer Genetics at McGill University.

Address correspondence to: Nicholas R. Bertos, 1160 Ave. des Pins O., Montreal, Quebec, Canada H3A 1A3. Phone: 514.398.2325; Fax: 514.398.6769; E-mail: nicholas.bertos@mcgill.ca.
1. Pinder SE, Ellis IO. The diagnosis and management of pre-invasive breast disease: ductal carcinoma in situ (DCIS) and atypical ductal hyperplasia (ADH)-current definitions and classification. Breast Cancer Res. 2003;5(5):254-257.

2. Li CI, Uribe DJ, Daling JR. Clinical characteristics of different histologic types of breast cancer. $\mathrm{BrJ}$ Cancer. 2005;93(9):1046-1052.

3. Weigelt B, Geyer FC, Reis-Filho JS. Histological types of breast cancer: how special are they? $\mathrm{Mol}$ Oncol. 2010;4(3):192-208

4. Arpino G, Clark GM, Mohsin S, Bardou VJ, Elledge RM. Adenoid cystic carcinoma of the breast: molecular markers, treatment, and clinical outcome. Cancer. 2002;94(8):2119-2127.

5. Pedersen L, Zedeler K, Holck S, Schiodt T, Mouridsen HT. Medullary carcinoma of the breast. Prevalence and prognostic importance of classical risk factors in breast cancer. Eur J Cancer. 1995; 31A(13-14):2289-2295

6. Diab SG, Clark GM, Osborne CK, Libby A, Allred DC, Elledge RM. Tumor characteristics and clinical outcome of tubular and mucinous breast carcinomas. J Clin Oncol. 1999;17(5):1442-1448.

7. Wolff AC, et al. American Society of Clinical Oncology/College of American Pathologists guideline recommendations for human epidermal growth factor receptor 2 testing in breast cancer. Arch Pathol Lab Med. 2007;131(1):18-43.

8. Jordan VC, Brodie AM. Development and evolution of therapies targeted to the estrogen receptor for the treatment and prevention of breast cancer. Steroids. 2007;72(1):7-25.

9. Patel RR, Sharma CG, Jordan VC. Optimizing the antihormonal treatment and prevention of breast cancer. Breast Cancer. 2007;14(2):113-122.

10. Bartlett JM, et al. Estrogen receptor and progesterone receptor as predictive biomarkers of response to endocrine therapy: a prospectively powered pathology study in the Tamoxifen and Exemestane Adjuvant Multinational trial. J Clin Oncol. 2011; 29(12):1531-1538.

11. Dowsett $M$, et al. Relationship between quantitative estrogen and progesterone receptor expression and human epidermal growth factor receptor 2 (HER-2) status with recurrence in the Arimidex, Tamoxifen, Alone or in Combination trial. J Clin Oncol. 2008;26(7):1059-1065.

12. Bardou VJ, Arpino G, Elledge RM, Osborne CK, Clark GM. Progesterone receptor status significantly improves outcome prediction over estrogen receptor status alone for adjuvant endocrine therapy in two large breast cancer databases. J Clin Oncol. 2003;21(10):1973-1979.

13. Stotter A, Walker R. Tumour markers predictive of successful treatment of breast cancer with primary endocrine therapy in patients over 70 years old: a prospective study. Crit Rev Oncol Hematol. 2010;75(3):249-256.

14. Stendahl M, Ryden L, Nordenskjold B, Jonsson PE, Landberg G, Jirstrom K. High progesterone receptor expression correlates to the effect of adjuvant tamoxifen in premenopausal breast cancer patients. Clin Cancer Res. 2006;12(15):4614-4618.

15. Junttila TT, et al. Ligand-independent HER2/ HER3/PI3K complex is disrupted by trastuzumab and is effectively inhibited by the PI3K inhibitor GDC-0941. Cancer Cell. 2009;15(5):429-440.

16. Clynes RA, Towers TL, Presta LG, Ravetch JV. Inhibitory $\mathrm{Fc}$ receptors modulate in vivo cytoxicity against tumor targets. Nat Med. 2000;6(4):443-446.

17. Slamon DJ, et al. Use of chemotherapy plus a monoclonal antibody against HER2 for metastatic breast cancer that overexpresses HER2. $N$ Engl J Med. 2001;344(11):783-792.

18. Smith I, et al. 2-year follow-up of trastuzumab after adjuvant chemotherapy in HER2-positive breast cancer: a randomised controlled trial. Lancet. 2007;369(9555):29-36.

19. Nishimura R, Arima N. Is triple negative a prognostic factor in breast cancer? Breast Cancer. 2008; 15(4):303-308.

20. Parise CA, Bauer KR, Brown MM, Caggiano V. Breast cancer subtypes as defined by the estrogen receptor (ER), progesterone receptor (PR), and the human epidermal growth factor receptor 2 (HER2) among women with invasive breast cancer in California, 1999-2004. Breast J. 2009;15(6):593-602.

21. Hammond ME, et al. American Society of Clinical Oncology/College of American Pathologists guideline recommendations for immunohistochemical testing of estrogen and progesterone receptors in breast cancer (unabridged version). Arch Pathol Lab Med. 2010;134(7):e48-e72.

22. Arslan C, Sari E, Aksoy S, Altundag K. Variation in hormone receptor and HER-2 status between primary and metastatic breast cancer: review of the literature. Expert Opin Ther Targets. 2011;15(1):21-30.

23. Ding L, et al. Genome remodelling in a basal-like breast cancer metastasis and xenograft. Nature. 2010;464(7291):999-1005.

24. Welsh AW, et al. Standardization of estrogen receptor measurement in breast cancer suggests false-negative results are a function of threshold intensity rather than percentage of positive cells. 
J Clin Oncol. 2011;29(22):2978-2984.

25. Shah SS, et al. Impact of American Society of Clinical Oncology/College of American Pathologists guideline recommendations on HER2 interpretation in breast cancer. Hum Pathol. 2010;41(1):103-106.

26. Torres L, Ribeiro FR, Pandis N, Andersen JA, Heim $\mathrm{S}$, Teixeira MR. Intratumor genomic heterogeneity in breast cancer with clonal divergence between primary carcinomas and lymph node metastases. Breast Cancer Res Treat. 2007;102(2):143-155.

27. Navin N, et al. Inferring tumor progression from genomic heterogeneity. Genome Res. 2010; 20(1):68-80

28. Navin N, et al. Tumour evolution inferred by single-cell sequencing. Nature. 2011;472(7341):90-94.

29. Hu Z, et al. The molecular portraits of breast tumors are conserved across microarray platforms. BMC Genomics. 2006;7:96.

30. Sorlie T, et al. Repeated observation of breast tumor subtypes in independent gene expression data sets. Proc Natl Acad Sci U S A. 2003;100(14):8418-8423.

31. Perou CM, et al. Molecular portraits of human breast tumours. Nature. 2000;406(6797):747-752.

32. Sorlie T, et al. Gene expression patterns of breast carcinomas distinguish tumor subclasses with clinical implications. Proc Natl Acad Sci U S A. 2001; 98(19):10869-10874.

33. Perou CM, et al. Distinctive gene expression patterns in human mammary epithelial cells and breast cancers. Proc Natl Acad Sci U S A. 1999;96(16):9212-9217.

34. Perreard L, et al. Classification and risk stratification of invasive breast carcinomas using a realtime quantitative RT-PCR assay. Breast Cancer Res. 2006;8(2):R23

35. Guedj M, et al. A refined molecular taxonomy of breast cancer [published online ahead of print July 25, 2011]. Oncogene. doi:10.1038/onc.2011.301.

36. Wirapati P, et al. Meta-analysis of gene expression profiles in breast cancer: toward a unified understanding of breast cancer subtyping and prognosis signatures. Breast Cancer Res. 2008;10(4):R65.

37. Reis-Filho JS, Weigelt B, Fumagalli D, Sotiriou C. Molecular profiling: moving away from tumor philately. Sci Transl Med. 2010;2(47):47ps43.

38. Sorlie T, et al. Repeated observation of breast tumor subtypes in independent gene expression data sets. Proc Natl Acad Sci U S A. 2003;100(14):8418-8423.

39. Parker JS, et al. Supervised risk predictor of breast cancer based on intrinsic subtypes. J Clin Oncol. 2009; 27(8):1160-1167.

40. Weigelt $B$, et al. Breast cancer molecular profiling with single sample predictors: a retrospective analysis. Lancet Oncol. 2010;11(4):339-349.

41. Mackay A, et al. Microarray-based class discovery for molecular classification of breast cancer: analysis of interobserver agreement. J Natl Cancer Inst. 2011; 103(8):662-673.

42. Nielsen TO, et al. Immunohistochemical and clinical characterization of the basal-like subtype of invasive breast carcinoma. Clin Cancer Res. 2004;10(16):5367-5374.

43. Carey LA, et al. The triple negative paradox: primary tumor chemosensitivity of breast cancer subtypes. Clin Cancer Res. 2007;13(8):2329-2334.

44. Perou CM, et al. Molecular portraits of human breast tumours. Nature. 2000;406(6797):747-752.

45. Hennessy BT, et al. Characterization of a naturally occurring breast cancer subset enriched in epithelial-to-mesenchymal transition and stem cell characteristics. Cancer Res. 2009;69(10):4116-4124.

46. Farmer $\mathrm{P}$, et al. Identification of molecular apocrine breast tumours by microarray analysis. Oncogene. 2005;24(29):4660-4671.

47. Lehmann BD, et al. Identification of human triple-negative breast cancer subtypes and preclinical models for selection of targeted therapies. J Clin Invest. 2011;121(7):2750-2767.

48. Natrajan R, et al. An integrative genomic and tran- scriptomic analysis reveals molecular pathways and networks regulated by copy number aberrations in basal-like, HER2 and luminal cancers. Breast Cancer Res Treat. 2010;121(3):575-589.

49. Bergamaschi A, et al. Distinct patterns of DNA copy number alteration are associated with different clinicopathological features and gene-expression subtypes of breast cancer. Genes Chromosomes Cancer. 2006;45(11):1033-1040.

50. Chin K, et al. Genomic and transcriptional aberrations linked to breast cancer pathophysiologies. Cancer Cell. 2006;10(6):529-541.

51. Hu X, et al. Genetic alterations and oncogenic pathways associated with breast cancer subtypes. Mol Cancer Res. 2009;7(4):511-522.

52. Adelaide J, et al. Integrated profiling of basal and luminal breast cancers. Cancer Res. 2007; 67(24):11565-11575

53. Korkola J, Gray JW. Breast cancer genomes--form and function. Curr Opin Genet Dev. 2010;20(1):4-14.

54. Jonsson G, et al. Genomic subtypes of breast cancer identified by array-comparative genomic hybridization display distinct molecular and clinical characteristics. Breast Cancer Res. 2010;12(3):R42.

55. van Wolfswinkel JC, Ketting RF. The role of small non-coding RNAs in genome stability and chromatin organization. J Cell Sci. 2010;123(pt 11):1825-1839.

56. Fabian MR, Sonenberg N, Filipowicz W. Regulation of mRNA translation and stability by microRNAs. Annu Rev Biochem. 2010;79:351-379.

57. Bockmeyer CL, et al. MicroRNA profiles of healthy basal and luminal mammary epithelial cells are distinct and reflected in different breast cancer subtypes [published online ahead of print March 17, 2011]. Breast Cancer Res Treat. doi:10.1007/ s10549-010-1303-3.

58. Blenkiron C, et al. MicroRNA expression profiling of human breast cancer identifies new markers of tumor subtype. Genome Biol. 2007;8(10):R214.

59. Adams BD, Guttilla IK, White BA. Involvement of microRNAs in breast cancer. Semin Reprod Med. 2008;26(6):522-536.

60. Enerly E, et al. miRNA-mRNA integrated analysis reveals roles for miRNAs in primary breast tumors. PLoS One. 2011;6(2):e16915.

61. Yu F, et al. let-7 regulates self renewal and tumorigenicity of breast cancer cells. Cell. 2007; 131(6):1109-1123.

62. Tavazoie SF, et al. Endogenous human microRNAs that suppress breast cancer metastasis. Nature. 2008; 451(7175):147-152.

63. Hurteau GJ, Carlson JA, Spivack SD, Brock GJ. Overexpression of the microRNA hsa-miR-200c leads to reduced expression of transcription factor 8 and increased expression of E-cadherin. Cancer Res. 2007;67(17):7972-7976.

64. Li XF, Yan PJ, Shao ZM. Downregulation of miR$193 \mathrm{~b}$ contributes to enhance urokinase-type plasminogen activator (uPA) expression and tumor progression and invasion in human breast cancer. Oncogene. 2009;28(44):3937-3948.

65. Van der Auwera I, Limame R, van Dam P, Vermeulen PB, Dirix LY, Van Laere SJ. Integrated miRNA and mRNA expression profiling of the inflammatory breast cancer subtype. Br J Cancer. 2010; 103(4):532-541.

66. Cheng C, Fu X, Alves P, Gerstein M. mRNA expression profiles show differential regulatory effects of microRNAs between estrogen receptor-positive and estrogen receptor-negative breast cancer. Genome Biol. 2009;10(9):R90.

67. Dedes KJ, et al. Down-regulation of the miRNA master regulators Drosha and Dicer is associated with specific subgroups of breast cancer. Eur J Cancer. 2011;47(1):138-150.

68. Grelier G, et al. Prognostic value of Dicer expression in human breast cancers and association with the mesenchymal phenotype. Br J Cancer. 2009;
101(4):673-683.

69. Lapidus RG, et al. Methylation of estrogen and progesterone receptor gene 5' CpG islands correlates with lack of estrogen and progesterone receptor gene expression in breast tumors. Clin Cancer Res. 1996;2(5):805-810.

70. Lapidus RG, et al. Mapping of ER gene CPG island methylation-specific polymerase chain reaction. Cancer Res. 1998;58(12):2515-2519.

71. Iwase $\mathrm{H}$, et al. DNA methylation analysis at distal and proximal promoter regions of the oestrogen receptor gene in breast cancers. Br J Cancer. 1999; 80(12):1982-1986.

72. Kamalakaran S, et al. DNA methylation patterns in luminal breast cancers differ from non-luminal subtypes and can identify relapse risk independent of other clinical variables. Mol Oncol. 2011; 5(1):77-92.

73. Ronneberg JA, et al. Methylation profiling with a panel of cancer related genes: association with estrogen receptor, TP53 mutation status and expression subtypes in sporadic breast cancer. $\mathrm{Mol}$ Oncol. 2011;5(1):61-76.

74. Smalley MJ, Reis-Filho JS, Ashworth A. BRCA1 and stem cells: tumour typecasting. Nat Cell Biol. 2008;10(4):377-379.

75. Liu S, et al. BRCA1 regulates human mammary stem/ progenitor cell fate. Proc Natl Acad Sci U S A. 2008; 105(5):1680-1685.

76. Prat A, Perou CM. Mammary development meets cancer genomics. Nat Med. 2009;15(8):842-844.

77. Lim E, et al. Aberrant luminal progenitors as the candidate target population for basal tumor development in BRCA1 mutation carriers. Nat Med. 2009; 15(8):907-913.

78. Molyneux G, et al. BRCA1 basal-like breast cancers originate from luminal epithelial progenitors and not from basal stem cells. Cell Stem Cell. 2010; $7(3): 403-417$

79. Visvader JE. Cells of origin in cancer. Nature. 2011; 469(7330):314-322.

80. Mani SA, et al. The epithelial-mesenchymal transition generates cells with properties of stem cells. Cell. 2008;133(4):704-715.

81. Kalluri R, Weinberg RA. The basics of epithelial-mesenchymal transition. J Clin Invest. 2009; 119(6):1420-1428.

82. Blick T, et al. Epithelial mesenchymal transition traits in human breast cancer cell lines. Clin Exp Metastasis. 2008;25(6):629-642.

83. Polyak K, Weinberg RA. Transitions between epithelial and mesenchymal states: acquisition of malignant and stem cell traits. Nat Rev Cancer. 2009; 9(4):265-273.

84. van der Pluijm G. Epithelial plasticity, cancer stem cells and bone metastasis formation. Bone. 2011; 48(1):37-43.

85. Weigelt B, Reis-Filho JS. Histological and molecular types of breast cancer: is there a unifying taxonomy? Nat Rev Clin Oncol. 2009;6(12):718-730.

86. Page DL. Special types of invasive breast cancer, with clinical implications. Am J Surg Pathol. 2003; 27(6):832-835.

87. Weigelt B, et al. Refinement of breast cancer classification by molecular characterization of histological special types. J Pathol. 2008;216(2):141-150.

88. Marchio C, et al. Genomic and immunophenotypical characterization of pure micropapillary carcinomas of the breast. J Pathol. 2008;215(4):398-410.

89. Marchio C, et al. Mixed micropapillary-ductal carcinomas of the breast: a genomic and immunohistochemical analysis of morphologically distinct components. J Pathol. 2009;218(3):301-315.

90. Lacroix-Triki M, et al. Mucinous carcinoma of the breast is genomically distinct from invasive ductal carcinomas of no special type. J Pathol. 2010; 222(3):282-298.

91. Weigelt B, Geyer FC, Horlings HM, Kreike B, Half- 
werk H, Reis-Filho JS. Mucinous and neuroendocrine breast carcinomas are transcriptionally distinct from invasive ductal carcinomas of no special type. Mod Pathol. 2009;22(11):1401-1414.

92. Persson M, Andren Y, Mark J, Horlings HM, Persson F, Stenman G. Recurrent fusion of MYB and NFIB transcription factor genes in carcinomas of the breast and head and neck. Proc Natl Acad Sci U S A. 2009;106(44):18740-18744

93. Tognon C, et al. Expression of the ETV6-NTRK3 gene fusion as a primary event in human secretory breast carcinoma. Cancer Cell. 2002;2(5):367-376

94. Tlsty TD, Coussens LM. Tumor stroma and regulation of cancer development. Annu Rev Pathol. 2006 1:119-150.

95. Bacac M, Provero P, Mayran N, Stehle JC, Fusco C, Stamenkovic I. A mouse stromal response to tumor invasion predicts prostate and breast cancer patient survival. PLoS One. 2006;1:e32.

96. Boersma BJ, et al. A stromal gene signature associated with inflammatory breast cancer. Int J Cancer. 2008; 122(6):1324-1332

97. Casey $\mathrm{T}$, et al. Molecular signatures suggest a major role for stromal cells in development of invasive breast cancer. Breast Cancer Res Treat. 2009; 114(1):47-62.

98. Farmer P, et al. A stroma-related gene signature predicts resistance to neoadjuvant chemotherapy in breast cancer. Nat Med. 2009;15(1):68-74.

99. Finak G, et al. Stromal gene expression predicts clinical outcome in breast cancer. Nat Med. 2008; 14(5):518-527.

100.Ma X-J, Dahiya S, Richardson E, Erlander M, Sgroi D. Gene expression profiling of the tumor microenvironment during breast cancer progression. Breast Cancer Research. 2009;11(1):R7.

101. Ghajar CM, Bissell MJ. Extracellular matrix control of mammary gland morphogenesis and tumorigenesis: insights from imaging. Histochem Cell Biol. 2008;130(6):1105-1118.

102. Tokes AM, et al. Stromal matrix protein expression following preoperative systemic therapy in breast cancer. Clin Cancer Res. 2009;15(2):731-739.

103. Provenzano PP, et al. Collagen density promotes mammary tumor initiation and progression. $B M C$ Med. 2008;6:11.

104. White DE, et al. Targeted disruption of beta1-integrin in a transgenic mouse model of human breast cancer reveals an essential role in mammary tumor induction. Cancer Cell. 2004;6(2):159-170.

105.Gudjonsson T, Ronnov-Jessen L, Villadsen R, Rank F, Bissell MJ, Petersen OW. Normal and tumorderived myoepithelial cells differ in their ability to interact with luminal breast epithelial cells for polarity and basement membrane deposition. J Cell Sci. 2002;115(pt 1):39-50.

106.Leek RD, Harris AL. Tumor-associated macrophages in breast cancer. J Mammary Gland Biol Neoplasia. 2002;7(2):177-189.

107. Lin EY, Gouon-Evans V, Nguyen AV, Pollard JW. The macrophage growth factor CSF-1 in mammary gland development and tumor progression. J Mammary Gland Biol Neoplasia. 2002;7(2):147-162.

108. Joyce JA, Pollard JW. Microenvironmental regulation of metastasis. Nat Rev Cancer. 2009;9(4):239-252.

109. Wyckoff J, et al. A paracrine loop between tumor cells and macrophages is required for tumor cell migration in mammary tumors. Cancer Res. 2004; 64(19):7022-7029.

110. Teschendorff AE, et al. Improved prognostic classification of breast cancer defined by antagonistic activation patterns of immune response pathway modules. BMC Cancer. 2010;10:604.

111. Oldford SA, Robb JD, Codner D, Gadag V, Watson $\mathrm{PH}$, Drover S. Tumor cell expression of HLA-DM associates with a Th1 profile and predicts improved survival in breast carcinoma patients. Int Immunol. 2006;18(11):1591-1602.

112. Marrogi AJ, et al. Study of tumor infiltrating lymphocytes and transforming growth factor-beta as prognostic factors in breast carcinoma. Int J Cancer. 1997;74(5):492-501.

113. Mahmoud SM, et al. Tumor-infiltrating CD8+ lymphocytes predict clinical outcome in breast cancer. J Clin Oncol. 2011;29(15):1949-1955.

114.Gobert $\mathrm{M}$, et al. Regulatory T cells recruited through CCL22/CCR4 are selectively activated in lymphoid infiltrates surrounding primary breast tumors and lead to an adverse clinical outcome. Cancer Res. 2009;69(5):2000-2009.

115.Gupta S, Joshi K, Wig JD, Arora SK. Intratumora FOXP3 expression in infiltrating breast carcinoma: Its association with clinicopathologic parameters and angiogenesis. Acta Oncol. 2007;46(6):792-797.

116. Bates GJ, et al. Quantification of regulatory T cells enables the identification of high-risk breast cancer patients and those at risk of late relapse. J Clin Oncol. 2006;24(34):5373-5380

117. Ladoire S, et al. Pathologic complete response to neoadjuvant chemotherapy of breast carcinoma is associated with the disappearance of tumor-infiltrating foxp3+ regulatory T cells. Clin Cancer Res. 2008; 14(8):2413-2420.

118. Rody A, et al. T-cell metagene predicts a favorable prognosis in estrogen receptor-negative and HER2 positive breast cancers. Breast Cancer Res. 2009; 11(2):R15.

119. Muller A, et al. Involvement of chemokine receptors in breast cancer metastasis. Nature. 2001 410(6824):50-56

120. Orimo A, Weinberg RA. Stromal fibroblasts in cancer: a novel tumor-promoting cell type. Cell Cycle. 2006; 5(15):1597-1601

121. Stuelten CH, DaCosta Byfield S, Arany PR, Karpova TS, Stetler-Stevenson WG, Roberts AB. Breast cancer cells induce stromal fibroblasts to express MMP-9 via secretion of TNF-alpha and TGF-beta. J Cell Sci. 2005;118(pt 10):2143-2153.

122.Trimboli AJ, et al. Pten in stromal fibroblasts suppresses mammary epithelial tumours. Nature. 2009;461(7267):1084-1091.

123. Nieto $Y$, et al. Prognostic analysis of tumour angiogenesis, determined by microvessel density and expression of vascular endothelial growth factor in high-risk primary breast cancer patients treated with high-dose chemotherapy. Br J Cancer. 2007; 97(3):391-397.

124. Uzzan B, Nicolas P, Cucherat M, Perret GY. Microvessel density as a prognostic factor in women with breast cancer: a systematic review of the literature and meta-analysis. Cancer Res. 2004;64(9):2941-2955.

125.Bernstein L, Ross RK. Endogenous hormones and breast cancer risk. Epidemiol Rev. 1993;15(1):48-65.

126. Dirat B, Bochet L, Escourrou G, Valet P, Muller C. Unraveling the obesity and breast cancer links: a role for cancer-associated adipocytes? Endocr Dev. 2010;19:45-52.

127. Dawood S, et al. Prognostic value of body mass index in locally advanced breast cancer. Clin Cancer Res. 2008;14(6):1718-1725.

128. McTiernan A, Irwin M, Vongruenigen V. Weight, physical activity, diet, and prognosis in breast and gynecologic cancers. J Clin Oncol. 2010; 28(26):4074-4080

129. Creighton CJ, et al. A gene transcription signature of obesity in breast cancer [published online ahead of print July 13, 2011]. Breast Cancer Res Treat. doi:10.1007/s10549-011-1595-y.

130.Shah SP, et al. Mutational evolution in a lobular breast tumour profiled at single nucleotide resolution. Nature. 2009;461(7265):809-813

131. Hess KR, Pusztai L, Buzdar AU, Hortobagyi GN. Estrogen receptors and distinct patterns of breast cancer relapse. Breast Cancer Res Treat. 2003; 78(1):105-118.

132. Kennecke H, et al. Metastatic behavior of breast cancer subtypes. J Clin Oncol. 2010;28(20):3271-3277.

133. Smid M, et al. Subtypes of breast cancer show preferential site of relapse. Cancer Res. 2008;68(9):3108-3114.

134.Pestalozzi BC, Brignoli S. Trastuzumab in CSF. J Clin Oncol. 2000;18(11):2349-2351.

135. Paget $\mathrm{S}$. The distribution of secondary growths in cancer of the breast. Lancet. 1889;133(3421):571-573.

136. Tabaries $S$, et al. Claudin-2 is selectively enriched in and promotes the formation of breast cancer liver metastases through engagement of integrin complexes. Oncogene. 2011;30(11):1318-1328.

137. Ouellet V, et al. CCN3 impairs osteoblast and stimulates osteoclast differentiation to favor breast cancer metastasis to bone. Am J Pathol. 2011; 178(5):2377-2388.

138. Oskarsson T, et al. Breast cancer cells produce tenascin $\mathrm{C}$ as a metastatic niche component to colonize the lungs. Nat Med. 2011;17(7):867-874.

139.Andre F, et al. CXCR4 expression in early breast cancer and risk of distant recurrence. Oncologist. 2009;14(12):1182-1188

140.Kang Y, et al. A multigenic program mediating breast cancer metastasis to bone. Cancer Cell. 2003; 3(6):537-549.

141.Kim MY, et al. Tumor self-seeding by circulating cancer cells. Cell. 2009;139(7):1315-1326.

142. Norton L, Massague J. Is cancer a disease of selfseeding? Nat Med. 2006;12(8):875-878

143. Comen E, Norton L, Massague J. Clinical implications of cancer self-seeding. Nat Rev Clin Oncol. 2011;8(6):369-377.

144.McAllister SS, et al. Systemic endocrine instigation of indolent tumor growth requires osteopontin. Cell. 2008;133(6):994-1005.

145.Gupta PB, et al. Systemic stromal effects of estrogen promote the growth of estrogen receptor-negative cancers. Cancer Res. 2007;67(5):2062-2071.

146. Punnoose EA, et al. Molecular biomarker analyses using circulating tumor cells. PLoS One. 2010; 5(9):e12517.

147. Munzone E, et al. Changes of HER2 status in circulating tumor cells compared with the primary tumor during treatment for advanced breast cancer. Clin Breast Cancer. 2010;10(5):392-397.

148.Aktas B, et al. Comparison of estrogen and progesterone receptor status of circulating tumor cells and the primary tumor in metastatic breast cancer patients. Gynecol Oncol. 2011;122(2):356-360.

149. Lu J, et al. Isolation of circulating epithelial and tumor progenitor cells with an invasive phenotype from breast cancer patients. Int J Cancer. 2010; 126(3):669-683

150.Aktas B, Tewes M, Fehm T, Hauch S, Kimmig R, Kasimir-Bauer S. Stem cell and epithelial-mesenchymal transition markers are frequently overexpressed in circulating tumor cells of metastatic breast cancer patients. Breast Cancer Res. 2009;11(4):R46. 